\title{
Evaluation of Naked Barley Landraces for Agro-morphological Traits
}

\author{
Ajaya Karkee ${ }^{\circledR}$, Krishna Hari Ghimire and Bal Krishna Joshi \\ ${ }^{1}$ National Agriculture Genetic Resources Center (National Genebank), NARC,Khumaltar, Nepal; @: \\ ajayakarkee@gmail.com; KHG: krishnahari.ghimire@yahoo.com; BKJ: joshibalak@yahoo.com
}

Received 23 Dec 2019, Revised 04 Jan 2020, Accepted 23 Feb 2020, Published

17 March 2020

Scientific Editors: Jiban Shrestha, Neena Amatya Gorkhali

Copyright $(\mathrm{C} 2020$ NARC. Permits unrestricted use, distribution and

reproduction in any medium provided the original work is properly cited.

The authors declare that there is no conflict of interest.

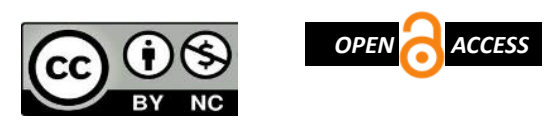

Licensed under the Creative Commons AttributionNonCommercial 4.0 International (CC BY-NC 4.0)

\section{ABSTRACT}

Naked barley (Hordeum vulgare var. nudum L.) is a traditional, culturally important, climate-resilient winter cereal crop of Nepal. Evaluation of the naked barely genotypes for yield and disease is fundamental for their efficient utilization in plant breeding schemes and effective conservation programs. Therefore, to identify high yielding and yellow rust resistant landraces of naked barley for hilly and mountainous agro-ecosystem, twenty naked barley landraces collected from different locations of Nepal, were evaluated in randomized complete block design (RCBD) with three replications during winter season of 2016 and 2017 at Khumaltar, Lalitpur, Nepal. Combined analysis of variances revealed that NGRC04902 (3.46 t/ha), NGRC00886 (3.28 t/ha), NGRC02309 (3.21 t/ha) and NGRC06026 (3.10 t/ha) were the high yielding landraces and statistically at par with the released variety 'Solu Uwa' (3.15 t/ha). The landraces namely NGRC00837 (ACI Value: 1.86) was found resistant to yellow rust diseases. Landraces NGRC06034 (131.7 days) and NGRC02363 (130.8 days) were found early maturing and NGRC02306 $(94.36 \mathrm{~cm})$ was found dwarf landraces among tested genotypes. These landraces having higher yield and better resistance to yellow rust need to be deployed to farmers' field to diversify the varietal options and used in resistant breeding program to improve the productivity of naked barley for Nepalese farmers.

Keywords: Hordeum vulgare var. nudum L., naked barley, resistant breeding, yellow rust

\section{सारांश}

उवा नेपालको एक परम्परागत तथा साँस्कृतिक महत्व भएको, जलवायु अनुकुलित हिउँदे वाली हो । उवाका रैथाने जातहरुको संरक्षण र बाली प्रजनन् मार्फत् दिगो उपयोगको लागि तिनीहरुको उत्पादकत्व तथा रोग अबरोधी क्षमता परीक्षण गर्न एक आधारभुत कार्य हो। त्यसैले, नेपालको पहाडी तथा हिमाली भेगको लागि उपयक्त हुने उवाका धेरै उत्पादन दिने र सिन्दुरे रोग अबरोधी उत्कृष्ट जातहरुको पहिचान गर्न विभिन्न स्थानबाट संकलन गरिएका बीस ओटा जातहरु समावेश गरी २०७३ र २०७४ सालको हिउँदमा ललितपुरको खुमलटारमा परीक्षण संचालन गरिएको थियो। परीक्षणको नतिजाले उवाका स्थानीय जातहरु NGRC04902 (३. ४६ मे.ट./हे), NGRC00886 (३.२६ मे.ट./हे), NGRC02309 (३. २१ मे.ट./हे) र NGRC06026 (३.१० मे.ट./हे) ले धेरै उत्पादन दिएको देखायो । स्थानीय जात NGRC00837 पहेलो सिन्दुरे रोग अबरोधी पाईयो । त्यसैगरी NGRC06034 (१३२ दिन) ₹ NGRC02363 (१३१ दिन) जात छिटो पाक्ने ₹ NGRC02306 ( $९$ से.मी.) अन्य जात भन्दा होचो पाईयो । यस अध्ययनबाट उवाका बढी उत्पादन दिने, चाँडै पाक्ने, होचो तथा पहेँलो सिन्दुरे रोग अबरोधी स्थानीय उत्कृष्ट जातहरु छनौट गरिएको छ। यी जातहरुलाई अकै व्यापक रुपमा कषकको खेतबारीमा थप परीक्षण गरी सिफारिस गरेमा उवाको उत्पादकत्व बढाउन सकिन्छ ।

\section{INTRODUCTION}

Barley (Hordeum vulgare L.) belongs to the genus Hordeum in the Triticaceae of Gramineae family. It is self-pollinated diploid $2 \mathrm{n}=14$. It is the fourth important cereal crop after wheat, maize and rice in the world (Akar et al 2009; Kandel et al 2019). There are two distinct forms of cultivated barley. The commonly grown barley (Hordeum vulgare L.) has intact husk cover of the caryopsis and the other form, known as naked or hullless barley (Hordeum vulgare var. nudum L.), has loose husk cover, 
which is easily separable upon threshing (Pandey et al 2009). This characteristic is controlled by a single gene locus ' $n u d$ ', the mutation of which occurred after domestication (Taketa et al 2008). Barley has historically been the prevalent cereal grown in marginal agricultural areas (Zohary and Hopf 2000) but the hullless barley cultivation is less common worldwide (Pandey et al 2009). Today, only a tiny fraction of world barley production is used directly for human consumption. It is usually grown either on land unsuitable for wheat cultivation, or where barley is preferred for cultural reasons (Fischbeck 2003). Ther is renewed interest in using naked barley as a component of human diets in western countries, arising from potential health benefits related to b-glucan content (Wood 2002) and low glycemic load (Foster-Powell et al 2002). In comparison to wheat, barley contains between four and six times to the levels of minerals such as calcium, magnesium, and potassium and ten times more iron (Ragaee et al 2006). Barley also has lower agronomic input requirements than wheat (Cross and Edwards-Jones 2006).

Naked barley is a traditional, culturally important, climate-resilient and highly nutritious winter cereal crop of the high mountainous region of Nepal (Ghimire et al 2019, Yadav et al 2018). The barley landraces from the Himalayas, particularly from the highlands of Nepal, share a significant part of world barley germplasm resources (Valkoun and Konopka 2004, Joshi et al 2017). In the highlands, its flour is mostly consumed as Satu (Flour prepared from roasted barley grains which can be eaten as it is and/or can be used in several cuisines), flat breads, dumplings and Thukpa (Tibetan noodle soup) and also mixed with flour of other crops like wheat, buckwheat and finger millet. Additionally, a special traditional fermented drink Chhyang and distilled liquor are prepared from the grains for different cultural and religious occasions by Lama and Sherpa tribes residing in mountainous region of Nepal (Gurung et al 2017, Palikhey et al 2017, Parajuli et al 2017, Pudasaini et al 2017). Yadav et al (2018) also found that the Shannon-Weaver diversity index (H') of naked barley ranged from 0.32 to 0.99 with a mean value of 0.73 , inferring tremendous diversity in the collection for the qualitative traits. Pandey et al (2009) evaluated hulless barley landraces of Nepal for resistance to Barley mild mosaic virus (BaYMV), powdery mildew and leaf rust and stated hulless barley germplasm of Nepal can be considered important source of resistance to BaYMV and powdery mildew but none of the genotypes show resistance to Puccinia hordei.

The crop is affected by many fungal, bacterial and viral diseases and amongst them the stripe rust (Puccinia striiformis f. sp. hordei) is the most important one (Selvakumar et al 2015) and occurred in Europe and Asia for many years and severe epidemics of the disease have been reported in northwestern and central European countries, India, Bangladesh, Nepal, China and Japan (Chen et al 1995) with the yield losses of 30-70\% (Dubin and Stubbs 1986). The highest grain yield loss of a susceptible barley cultivar was found to be $72 \%$ in the US (Marshall and Sutton 1995) and stripe rust greatly reduces malting quality (Line 2002).

Genetic variability of naked barley genotypes is necessary for its genetic improvement and landraces are more diverse than modern varieties for a number of traits (Joshi et al 2019). The knowledge on naked barley genetic variability and its potential yield as well as yield associate traits is important for further its use in breeding programs. Therefore, we conducted this research as a pre-breeding activity of genebank accessions with the objective of identifying high yielding and stripe rust resistant naked barley landraces for hills of Nepal.

\section{MATERIALS AND METHODS}

\section{Plant materials and site description}

This study used 20 naked barley genotypes including 19 landraces collected from 13 different districts of Nepal (Figure 1) and a released variety Solu Uwa as a check (Table 1 (Joshi et al 2017b for detail of Solu Uwa). These genotypes were selected based on their performance during preliminary characterization at Genebank, Khumaltar. The research field of National Agriculture Genetic Resource Centre is located at an altitude of $1368 \mathrm{~m}$, latitude of $27^{\circ} 40^{\prime} \mathrm{N}$ and longitude of $085^{\circ} 20^{\prime} \mathrm{E}$ (Genebank, 2018). Soil type of the experimental blocks was black and loamy (Ghimire and Magar 2017, Ghimire et al 2013). 


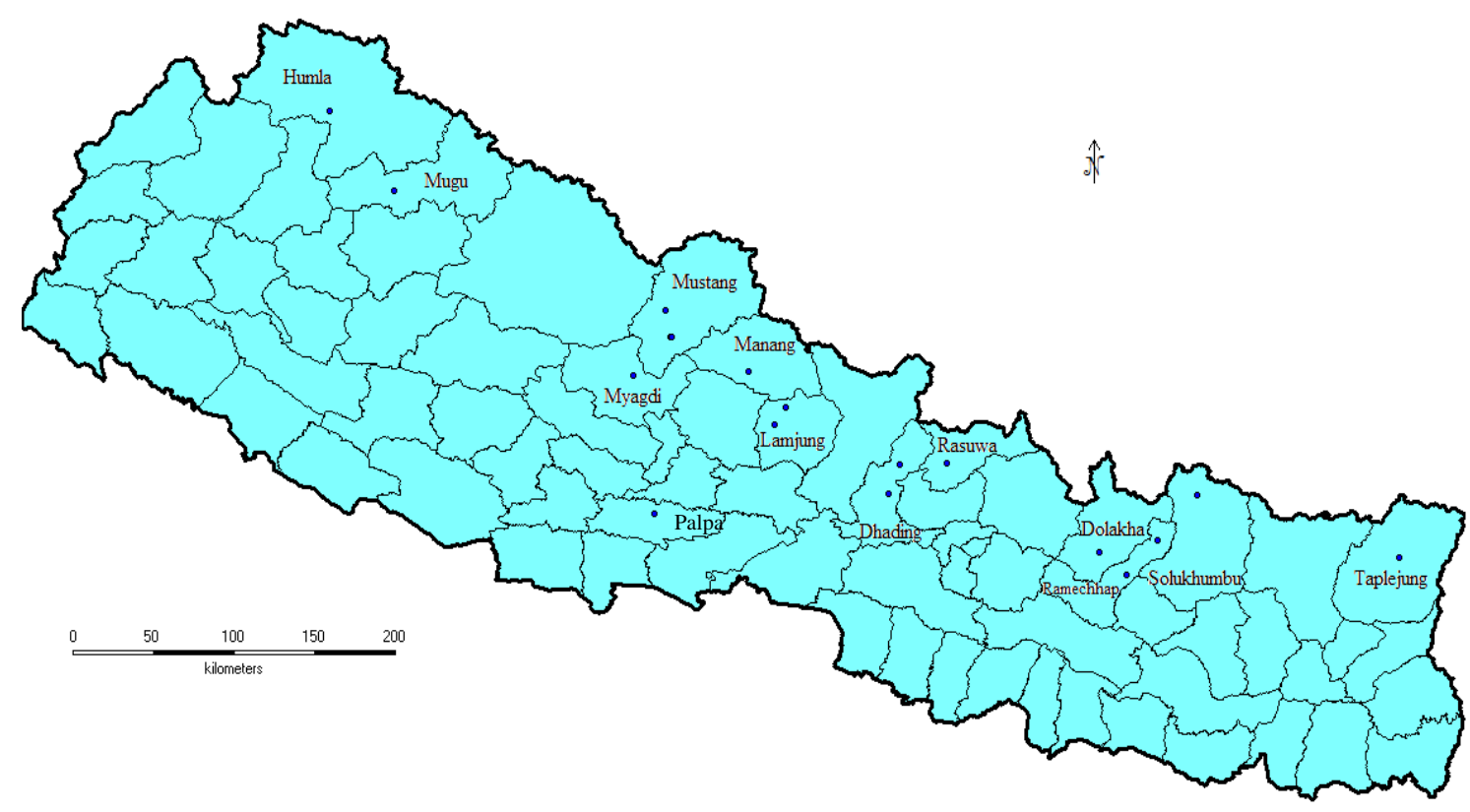

Figure 1. Map of Nepal showing collection sites of test landraces.

Table 1. Detail of landraces evaluated in Khumaltar during winter of 2016 and 2017

\begin{tabular}{rllll}
\hline SN & Accession no & Local name & Collection district & Altitude (m) \\
\hline 1 & NGRC00837 & Karu & Palpa & 780 \\
\hline 2 & NGRC00886 & Karu & Myagdi, Chitre & 2300 \\
\hline 3 & NGRC02306 & Uwa & Mustang, Chhoser & 3800 \\
\hline 4 & NGRC02309 & Uwa & Solukhumbu, Janshesi & 2670 \\
\hline 5 & NGRC02312 & Uwa & Dhading, Khadin & 2200 \\
\hline 6 & NGRC02318 & Uwa & Taplejung, Tanku & 1900 \\
\hline 7 & NGRC02350 & Magheuwa & Ramechhap, Sano balding & 2100 \\
\hline 8 & NGRC02357 & Kalouwa & Mustang, Jarkot & 3353 \\
\hline 9 & NGRC02363 & Kalouwa & Rasuwa, Chilime & 2043 \\
\hline 10 & NGRC04003 & Jau & Mustang, Dhumba & 2800 \\
\hline 11 & NGRC04894 & Jau & Humla, Danda Phaya & 3500 \\
\hline 12 & NGRC04902 & Local Uwa & Manang, Pisang & 3697 \\
\hline 13 & NGRC04903 & JhuseUwa & Mugu, Kartikswarda & 2200 \\
\hline 14 & NGRC06024 & Uwa & Mustang, Marpha & 2695 \\
\hline 15 & NGRC06026 & Jau & Mustang, Khionga & 2690 \\
\hline 16 & NGRC06034 & Uwa & Dhading, Jharlang & 1085 \\
\hline 17 & NGRC06036 & Local Uwa & Ramechhap, Gumdel & 2500 \\
\hline 18 & NGRC06042 & Uwa & Lamjung, Taghring & 2713 \\
\hline 19 & NGRC06043 & Local Uwa & Lamjung, Ghanapokhara & 1741 \\
\hline 20 & Solu $U w a$ & Released check & Dolakha, Mainapokhari & 1740 \\
\hline & & & & \\
\hline
\end{tabular}

\section{Field experiments}

The experiments were conducted in a randomized complete block design (RCBD) with 3 replications during 2016/17 and 2017/18 at NAGRC Khumaltar, Lalitpur. Sowing was done on November 24 and December 6 during 2016 and 2017 respectively. Each entry was sown continuously in eight rows of $3 \mathrm{~m}$ length with distance of $25 \mathrm{~cm}$ between rows. The field was fertilized with Organic manure at the rate of $6 \mathrm{t} / \mathrm{ha}$ during land preparation and NPK at the rate of 100:50:0 kg/ha supplied from DAP and Urea during sowing time as a basal application. Weeding was done as per required at the field and irrigation was done at critical stage of irrigation i.e. tillering, bolding and flowering stage. Five plants were randomly selected in each plot and tagged in an advance for the convenience of recording observations. 


\section{Data collection and data analysis}

Agronomic characteristics such as days to heading and maturity, plant height $(\mathrm{cm})$, number of grains per spike, number of spikes per $\mathrm{m}^{2}$, thousand kernel weight $(\mathrm{g})$ and grain yield $(\mathrm{kg} / \mathrm{ha})$ were recorded following IPGRI descriptors (IPGRI 1994). Thousand kernel weight and grain yield were recorded after sun-drying in gram per plot and later extrapolated to kilogram $(\mathrm{kg})$ per hectare.

Percent yellow rust diseases severity was recorded before crop maturity to the modified Cobb's scale (Peterson et al. 1948) and reaction based on Roelfs et al (1992). Coefficient of infection (CI) was calculated by multiplying of diseases severity (DS) and constant values of infection type (IF). The constant values for infection types were used based on: R 1/4 0.1, MR 1/4 0.25, M 1/4 0.5, MS 1/4 0.75 and $\mathrm{S} 1 / 41$ (Pathan and Park 2006).

Statistical analysis of data for yield components and average coefficient of infection (ACI) carried out by MSTAT-C software version 1.3 (computer-based statistical software developed by the crop and Soil Sciences, Department of Michigan State University, USA). Analysis of variance (ANOVA) was performed using General Linear Model (Gomez and Gomez 1984) and comparison of genotypes was made based on Duncan's multiple range tests at 5\% level of significance (Duncan 1955, Shrestha 2019).

\section{RESULTS}

ANOVA was performed for days to heading and maturity, plant height, number of grains per spike; number of spikes per $\mathrm{m}^{2}, 1000$ kernel weight (TKW), grain yield and average coefficient of infection (ACI) for yellow rust of 20 tested genotypes for two years as well as combined over years.

Very highly significant differences $(\mathrm{p}<0.0001)$ were observed among tested landraces for days to heading and maturity in 2016 and 2017 (Table 2). Very highly significant differences $(p<0.001)$ were found among tested landraces for plant height in 2016 but non-significant in 2017 (Table 3). Likewise, Average coefficient of infection for yellow rust was found very highly significant $(\mathrm{p}<0.0001)$ different among tested landraces in both years (Table 3). Highly significant differences $(\mathrm{p}<0.001)$ were observed among tested landraces for spikes per $\mathrm{m}^{2}$ in 2016 but non-significant in 2017 and Very highly significant were found in grains per spike among the tested genotypes in both years (Table 4). TKW and grain yield were found very highly significant differences $(\mathrm{p}<0.0001)$ among tested landraces in both years (Table 4).

Combined over year data showed very highly significant differences for days to heading $(\mathrm{p}<0.0001)$, days to maturity $(\mathrm{p}<0.0001)$ (Table 2$)$, ACI value for yellow rust $(\mathrm{p}<0.0001)$ (Table-3), grains per spike $(\mathrm{p}<0.0001)$ (Table 4), TKW $(\mathrm{p}<0.001)$ and grain yield $(\mathrm{p}<0.0001)$ (Table 5) where only significant difference between tested genotypes found in plant height $(\mathrm{p}<0.01)$ (Table 3) and highly significant different between tested genotypes found in spikes per $\mathrm{m}^{2}(\mathrm{p}<0.001)$ (Table 4).

Landraces NGRC06034 and NGRC02363 were found the earliest for heading and maturity respectively. Plant height of landraces NGRC02306 and NGRC02312 were found the shortest and longest respectively among the tested genotypes. Genotypes NGRC00837 was found significantly lower ACI value than check variety, Solu Uwa (8.03). Grains per spike of ten out of twenty tested landraces were found higher than overall mean (58.56), TKW (g) and grain yield (t/ha) of twelve out of twenty tested landraces were found higher than overall mean $(38.11 \mathrm{~g})$ and $(2.81 \mathrm{t} / \mathrm{ha})$ respectively. Grain yield of landraces NGRC4902 (3.46 t/ha), NGRC00886 (3.28 t/ha), NGRC02309 (3.21 t/ha) were found at par but higher than check variety Solu Uwa $(3.15 \mathrm{t} / \mathrm{ha})$.

Genotype $\times$ year $(\mathrm{G} \times \mathrm{E})$ interaction was non-significant for days to heading (Table 2), days to maturity (Table 2), plant height (Table 3), grain per spikes (Table 4), spikes per $\mathrm{m}^{2}$ (Table 4) TKW (Table 5) and grain yield (Table 5) but significant different for average coefficient of infection (ACI) for yellow rust (Table 3) among the year. Yield and yield attributing traits differed significantly with each other in the tested years but non-significant interaction between genotypes and growing years indicated the stable performance of tested genotypes. 
Table 2. Mean days to heading and maturity of naked barley landraces over the year

\begin{tabular}{|c|c|c|c|c|c|c|}
\hline \multirow[t]{2}{*}{ Genotype } & \multicolumn{3}{|c|}{ Days to heading } & \multicolumn{3}{|c|}{ Days to maturity } \\
\hline & 2016 & 2017 & Combined & 2016 & 2017 & Combined \\
\hline NGRC00837 & $93.33^{\mathrm{ij}}$ & $86.67^{\mathrm{j}}$ & $90.00^{\mathrm{kl}}$ & $133.7^{\mathrm{f}}$ & $131.3^{\text {efg }}$ & $132.5^{\mathrm{ijk}}$ \\
\hline NGRC00886 & $91.33^{\mathrm{jk}}$ & $88.33^{\mathrm{ij}}$ & $89.83^{\mathrm{kl}}$ & $135.0^{\mathrm{ef}}$ & $134.0^{\text {de }}$ & $134.5^{\mathrm{hi}}$ \\
\hline NGRC02306 & $99.00^{\mathrm{de}}$ & $93.33^{\text {ef }}$ & $96.17^{\mathrm{d}}$ & $146.3^{b}$ & $144.0^{\mathrm{ab}}$ & $145.2^{\mathrm{bc}}$ \\
\hline NGRC02309 & $101.00^{\mathrm{cd}}$ & $98.00^{\mathrm{bc}}$ & $99.50^{b c}$ & $144.0^{\mathrm{bc}}$ & $136.3^{\mathrm{d}}$ & $140.2^{\mathrm{def}}$ \\
\hline NGRC02312 & $97.33^{\mathrm{ef}}$ & $92.67^{\mathrm{ef}}$ & $95.00^{\mathrm{de}}$ & $143.0^{\mathrm{bcd}}$ & $135.7^{\mathrm{d}}$ & $139.3^{\text {efg }}$ \\
\hline NGRC02318 & $96.33^{\mathrm{fg}}$ & $92.00^{\mathrm{fg}}$ & $94.17^{\text {efg }}$ & $140.7^{\mathrm{cd}}$ & $136.0^{\mathrm{d}}$ & $138.3^{\mathrm{dg}}$ \\
\hline NGRC02350 & $94.67^{\mathrm{ghi}}$ & $90.00^{\mathrm{ghi}}$ & $92.33^{\text {hij }}$ & $135.0^{\mathrm{ef}}$ & $132.7^{\mathrm{e}}$ & $133.8^{\mathrm{ij}}$ \\
\hline NGRC02357 & $105.3^{\mathrm{ab}}$ & $102.0^{\mathrm{a}}$ & $103.7^{\mathrm{a}}$ & $151.3^{\mathrm{a}}$ & $146.7^{\mathrm{a}}$ & $149.0^{\mathrm{a}}$ \\
\hline NGRC02363 & $96.00^{\text {fgh }}$ & $90.33^{\mathrm{ghi}}$ & $93.17^{\text {fgh }}$ & $132.7^{\mathrm{f}}$ & $129.0^{\mathrm{g}}$ & $130.8^{\mathrm{k}}$ \\
\hline NGRC04003 & $101.7^{\mathrm{c}}$ & $96.00^{\mathrm{cd}}$ & $98.83^{c}$ & $146.3^{b}$ & $136.3^{\mathrm{d}}$ & $141.3^{\mathrm{de}}$ \\
\hline NGRC04894 & $107.0^{\mathrm{a}}$ & $102.00^{\mathrm{a}}$ & $104.5^{\mathrm{a}}$ & $146.3^{\mathrm{b}}$ & $146.0^{\mathrm{ab}}$ & $146.2^{\mathrm{b}}$ \\
\hline NGRC04902 & $96.00^{\text {fgh }}$ & $93.00^{\mathrm{ef}}$ & $94.50^{\mathrm{def}}$ & $146.3^{b}$ & $143.3^{\mathrm{b}}$ & $144.8^{\mathrm{bc}}$ \\
\hline NGRC04903 & $92.33^{\mathrm{ij}}$ & $89.33^{\mathrm{hi}}$ & $90.83^{\mathrm{jk}}$ & $133.3^{\mathrm{f}}$ & $132.3^{\text {ef }}$ & $132.8^{\mathrm{ijk}}$ \\
\hline NGRC06024 & $103.0^{\mathrm{bc}}$ & $98.67^{b}$ & $100.8^{b}$ & $145.0^{\mathrm{bc}}$ & $145.0^{\mathrm{ab}}$ & $145.0^{\mathrm{bc}}$ \\
\hline NGRC06026 & $96.00^{\mathrm{fgh}}$ & $94.33^{\mathrm{de}}$ & $95.17^{\mathrm{de}}$ & $145.7^{b}$ & $139.3^{c}$ & $142.5^{\mathrm{cd}}$ \\
\hline NGRC06034 & $88.33^{1}$ & $84.00^{\mathrm{k}}$ & $86.17^{\mathrm{m}}$ & $133.7^{\mathrm{f}}$ & $129.7^{\mathrm{fg}}$ & $131.7^{\mathrm{jk}}$ \\
\hline NGRC06036 & $93.67^{\text {hij }}$ & $88.33^{\mathrm{ij}}$ & $91.00^{\mathrm{ijk}}$ & $135.3^{\text {ef }}$ & $132.7^{\mathrm{e}}$ & $134.0^{\mathrm{ij}}$ \\
\hline NGRC06042 & $94.00^{\mathrm{ghi}}$ & $91.33^{\text {fgh }}$ & $92.67^{\text {ghi }}$ & $133.7^{\mathrm{f}}$ & $132.3^{\text {ef }}$ & $133.0^{\mathrm{ijk}}$ \\
\hline NGRC06043 & $96.00^{\text {fgh }}$ & $92.00^{\mathrm{fg}}$ & $94.00^{\text {efgh }}$ & $138.7^{\mathrm{de}}$ & $135.7^{\mathrm{d}}$ & $137.2^{\mathrm{gh}}$ \\
\hline Solu Uwa & $89.33^{\mathrm{kl}}$ & $88.67^{\mathrm{ij}}$ & $89.00^{1}$ & $135.0^{\mathrm{ef}}$ & $132.0^{\mathrm{ef}}$ & $133.5^{\mathrm{ijk}}$ \\
\hline $\mathrm{P}$ value & $<0.0001$ & $<0.0001$ & $<0.0001$ & $<0.0001$ & $<0.0001$ & $<0.0001$ \\
\hline $\begin{array}{l}\mathrm{P} \text { value } \\
\text { year×genotype }\end{array}$ & & & 0.103 & & & 0.05 \\
\hline $\operatorname{LSD}(0.05)$ & 2.639 & 2.241 & 1.703 & 4.586 & 2.906 & 2.671 \\
\hline $\mathrm{CV}, \%$ & 1.65 & 1.46 & 1.57 & 1.98 & 1.29 & 1.68 \\
\hline
\end{tabular}

Table 3. Mean plant height and average coefficient of infection of naked barley landraces over the year Genotype

Plant height $(\mathrm{cm})$

\begin{tabular}{|c|c|c|c|c|c|c|}
\hline & & \\
\hline & 2016 & 2017 & Combined & 2016 & 2017 & Combined \\
\hline NGRC00837 & $112.9^{\mathrm{ef}}$ & $91.88^{\mathrm{b}}$ & $104.4^{\mathrm{bc}}$ & $2.06^{\mathrm{e}}$ & $2.94^{\mathrm{efgh}}$ & $1.86^{\mathrm{e}}$ \\
\hline NGRC00886 & $113.4^{\mathrm{de}}$ & $110.9^{b}$ & $112.2^{\mathrm{bc}}$ & $48.91^{\mathrm{a}}$ & $24.28^{\mathrm{abc}}$ & $34.16^{\mathrm{ab}}$ \\
\hline NGRC02306 & $98.47^{\mathrm{k}}$ & $90.25^{b}$ & $94.36^{\mathrm{c}}$ & $13.07^{\mathrm{cd}}$ & $1.77^{\text {fgh }}$ & $4.99^{\mathrm{de}}$ \\
\hline NGRC02309 & $123.5^{\mathrm{a}}$ & $100.0^{\mathrm{b}}$ & $111.8^{b c}$ & $32.46^{\mathrm{abc}}$ & $14.96^{\mathrm{abcde}}$ & $21.87^{b c}$ \\
\hline NGRC02312 & $116.6^{\mathrm{bc}}$ & $175.1^{\mathrm{a}}$ & $145.8^{\mathrm{a}}$ & $87.36^{\mathrm{a}}$ & $39.45^{\mathrm{a}}$ & $58.15^{\mathrm{a}}$ \\
\hline NGRC02318 & $103.1^{\mathrm{ij}}$ & $91.53^{\mathrm{b}}$ & $97.33^{b c}$ & $64.68^{a}$ & $35.60^{\mathrm{a}}$ & $57.56^{\mathrm{a}}$ \\
\hline NGRC02350 & $120.9^{a}$ & $114.0^{\mathrm{b}}$ & $117.5^{b}$ & $58.56^{\mathrm{a}}$ & $25.31^{\mathrm{abc}}$ & $38.25^{\mathrm{ab}}$ \\
\hline NGRC02357 & $117.2^{\mathrm{b}}$ & $101.3^{\mathrm{b}}$ & $109.3^{b c}$ & $7.17^{\mathrm{d}}$ & $5.55^{\text {cdefg }}$ & $5.82^{\mathrm{de}}$ \\
\hline NGRC02363 & $108.9^{\mathrm{g}}$ & $105.4^{\mathrm{b}}$ & $107.1^{b c}$ & $56.83^{\mathrm{a}}$ & $13.59^{\mathrm{abcde}}$ & $27.79^{\mathrm{ab}}$ \\
\hline NGRC04003 & $107.1^{\mathrm{gh}}$ & $98.85^{b}$ & $103.0^{\mathrm{bc}}$ & $76.71^{\mathrm{a}}$ & $10.36^{\text {abcdef }}$ & $28.37^{\mathrm{ab}}$ \\
\hline NGRC04894 & $107.6^{\mathrm{gh}}$ & $88.91^{\mathrm{b}}$ & $98.26^{b c}$ & $50.91^{\mathrm{a}}$ & $0.77^{\mathrm{gh}}$ & $8.49^{\mathrm{cd}}$ \\
\hline NGRC04902 & $114.3^{\text {bcde }}$ & $105.8^{\mathrm{b}}$ & $110.0^{\mathrm{bc}}$ & $78.26^{\mathrm{a}}$ & $12.33^{\mathrm{a}}$ & $52.52^{\mathrm{ab}}$ \\
\hline NGRC04903 & $113.7^{\text {cde }}$ & $102.5^{b}$ & $108.1^{b c}$ & $46.06^{\mathrm{ab}}$ & $17.92^{\mathrm{abcd}}$ & $28.67^{\mathrm{ab}}$ \\
\hline NGRC06024 & $105.2^{\mathrm{hi}}$ & $95.77^{b}$ & $100.5^{\mathrm{bc}}$ & $15.03^{\mathrm{bcd}}$ & $000^{\mathrm{h}}$ & $2.86^{\mathrm{de}}$ \\
\hline NGRC06026 & $109.9^{\mathrm{fg}}$ & $89.11^{\mathrm{b}}$ & $99.49^{b c}$ & $8.76^{\mathrm{d}}$ & $4.81^{\text {defg }}$ & $6.17^{\mathrm{d}}$ \\
\hline NGRC06034 & $121.5^{\mathrm{a}}$ & $115.1^{\mathrm{b}}$ & $118.3^{b}$ & $62.80^{\mathrm{a}}$ & $29.57^{\mathrm{ab}}$ & $42.82^{\mathrm{ab}}$ \\
\hline NGRC06036 & $117.3^{\mathrm{b}}$ & $112.9^{\mathrm{b}}$ & $115.1^{\mathrm{bc}}$ & $79.04^{\mathrm{a}}$ & $26.94^{\mathrm{abc}}$ & $45.99^{\mathrm{ab}}$ \\
\hline NGRC06042 & $116.3^{\mathrm{bcd}}$ & $108.2^{\mathrm{b}}$ & $112.3^{b c}$ & $52.98^{a}$ & $42.38^{\mathrm{a}}$ & $46.94^{\mathrm{ab}}$ \\
\hline NGRC06043 & $101.3^{\mathrm{jk}}$ & $93.94^{\mathrm{b}}$ & $97.64^{\mathrm{bc}}$ & $45.15^{\mathrm{ab}}$ & $33.12^{\mathrm{a}}$ & $38.25^{\mathrm{ab}}$ \\
\hline Solu Uwa & $121.0^{\mathrm{a}}$ & $95.90^{\mathrm{b}}$ & $108.5^{b c}$ & $10.80^{\mathrm{cd}}$ & $6.61^{\text {bcdefg }}$ & $8.03^{\mathrm{d}}$ \\
\hline $\mathrm{P}$ value & $<0.0001$ & 0.17 & 0.02 & $<0.0001$ & $<0.0001$ & $<0.0001$ \\
\hline $\begin{array}{l}\mathrm{P} \text { value } \\
\text { year×genotype }\end{array}$ & & & 0.34 & & & 0.019 \\
\hline $\operatorname{LSD}(0.05)$ & 3.14 & 44.91 & 22.68 & 3.07 & 3.32 & 1.48 \\
\hline $\mathrm{CV}, \%$ & 5.59 & 26.03 & 18.19 & 19.50 & 34.82 & 26.18 \\
\hline
\end{tabular}


Table 4. Mean spikes per $\mathbf{m}^{2}$ and grain per spikes of naked barley landraces over the year

\begin{tabular}{|c|c|c|c|c|c|c|}
\hline \multirow[t]{2}{*}{ Genotype } & \multicolumn{3}{|c|}{ Spikes/per } & \multicolumn{3}{|c|}{ Grain/spike, n } \\
\hline & 2016 & 2017 & Combined & 2016 & 2017 & Combined \\
\hline NGRC00837 & $183.7^{\mathrm{abc}}$ & $244.7^{\mathrm{abc}}$ & $214.2^{\text {abcdef }}$ & $64.80^{\mathrm{bc}}$ & $56.27^{\text {cdef }}$ & $60.53^{c}$ \\
\hline NGRC00886 & $143.7^{\text {cdefg }}$ & $306.7^{\mathrm{ab}}$ & $225.2^{\text {abcde }}$ & $61.47^{\text {cdef }}$ & $58.20^{\mathrm{bcd}}$ & $59.83^{c}$ \\
\hline NGRC02306 & $144.7^{\text {cdefg }}$ & $175.7^{\mathrm{c}}$ & $160.2^{\text {fg }}$ & $57.20^{\mathrm{def}}$ & $49.60^{\text {fgh }}$ & $53.40^{\mathrm{EF}}$ \\
\hline NGRC02309 & $206.0^{\mathrm{a}}$ & $297.3^{\mathrm{ab}}$ & $251.7^{\mathrm{a}}$ & $63.67^{\mathrm{bcd}}$ & $58.13^{\mathrm{bcd}}$ & $60.90^{C}$ \\
\hline NGRC02312 & $142.3^{\text {defg }}$ & $237.7^{\mathrm{abc}}$ & $190.0^{\text {bcdefg }}$ & $78.60^{\mathrm{a}}$ & $71.60^{\mathrm{a}}$ & $75.10^{\mathrm{A}}$ \\
\hline NGRC 02318 & $145.7^{\text {cdefg }}$ & $219.0^{\mathrm{abc}}$ & $182.3^{\text {defg }}$ & $70.13^{b}$ & $64.73^{\mathrm{ab}}$ & $67.43^{\mathrm{b}}$ \\
\hline NGRC02350 & $118.7^{\mathrm{g}}$ & $169.7^{\mathrm{c}}$ & $144.2^{\mathrm{g}}$ & $56.20^{\mathrm{ef}}$ & $59.67^{\text {bcd }}$ & $57.93^{\text {cde }}$ \\
\hline NGRC02357 & $179.0^{\mathrm{abcd}}$ & $322.7^{\mathrm{a}}$ & $150.8^{\mathrm{g}}$ & $56.73^{\mathrm{def}}$ & $47.93^{\mathrm{gh}}$ & $52.33^{\mathrm{f}}$ \\
\hline NGRC02363 & $125.0^{\mathrm{fg}}$ & $216.7^{\mathrm{abc}}$ & $170.8^{\text {efg }}$ & $63.60^{\mathrm{bcd}}$ & $55.00^{\text {cdefg }}$ & $59.30^{\mathrm{cd}}$ \\
\hline NGRC04003 & $169.7^{\text {abcdef }}$ & $214.7^{\mathrm{abc}}$ & $192.2^{\text {bcdefg }}$ & $58.67^{\text {cdef }}$ & $56.53^{\text {cdef }}$ & $57.60^{\text {cde }}$ \\
\hline NGRC04894 & $180.7^{\mathrm{abcd}}$ & $176.7^{\mathrm{c}}$ & $178.7^{\text {defg }}$ & $55.53^{\mathrm{f}}$ & $44.60^{\mathrm{h}}$ & $49.90^{f}$ \\
\hline NGRC04902 & $161.3^{\text {bcdef }}$ & $319.3^{\mathrm{ab}}$ & $240.3^{\mathrm{abc}}$ & $55.53^{\mathrm{f}}$ & $53.40^{\text {defg }}$ & $54.47^{\text {def }}$ \\
\hline NGRC04903 & $150.7^{\text {cdefg }}$ & $217.3^{\mathrm{abc}}$ & $184.0^{\text {cdefg }}$ & $63.40^{\mathrm{bcd}}$ & $60.80^{\mathrm{bc}}$ & $62.10^{c}$ \\
\hline NGRC06024 & $166.0^{\text {abcdef }}$ & $302.0^{\mathrm{ab}}$ & $234.0^{\mathrm{abcd}}$ & $56.40^{\mathrm{ef}}$ & $44.40^{\mathrm{h}}$ & $50.40^{f}$ \\
\hline NGRC06026 & $148.7^{\text {cdefg }}$ & $317.7^{\mathrm{ab}}$ & $233.2^{\text {abcd }}$ & $55.67^{\mathrm{f}}$ & $53.27^{\text {defg }}$ & $54.47^{\mathrm{def}}$ \\
\hline NGRC06034 & $147.3^{\text {cdefg }}$ & $249.3^{\mathrm{abc}}$ & $198.3^{\text {abcdefg }}$ & $63.53^{\mathrm{bcd}}$ & $55.87^{\text {cdef }}$ & $59.70^{c}$ \\
\hline NGRC06036 & $131.7^{\text {efg }}$ & $200.3^{b c}$ & $166.0^{\mathrm{fg}}$ & $59.87^{\text {cdef }}$ & $57.93^{\mathrm{bcd}}$ & $58.90^{\mathrm{cd}}$ \\
\hline NGRC06042 & $197.3^{\mathrm{ab}}$ & $323.3^{\mathrm{a}}$ & $160.3^{\mathrm{fg}}$ & $64.93^{\mathrm{bc}}$ & $50.27^{\text {efgh }}$ & $57.60^{\text {cde }}$ \\
\hline NGRC06043 & $129.7^{\mathrm{efg}}$ & $223.7^{\mathrm{abc}}$ & $176.7^{\text {efg }}$ & $58.53^{\text {cdef }}$ & $56.93^{56.93}$ & $57.73^{\text {cde }}$ \\
\hline Solu Uwa & $166.3^{\text {abcdef }}$ & $319.3^{\mathrm{ab}}$ & $242.0^{\mathrm{ab}}$ & $62.80^{\text {cde }}$ & $56.33^{\text {cdef }}$ & $59.57^{\mathrm{c}}$ \\
\hline $\mathrm{P}$ value & 0.024 & 0.06 & 0.029 & $<0.0001$ & $<0.0001$ & $<0.0001$ \\
\hline $\begin{array}{l}\text { P value } \\
\text { yearxgenotype }\end{array}$ & & & 0.38 & & & 0.12 \\
\hline $\operatorname{LSD}(0.05)$ & 41.04 & 119.3 & 56.50 & 6.965 & 7.248 & 4.945 \\
\hline $\mathrm{CV}, \%$ & 15.82 & 28.57 & 26.36 & 6.87 & 7.89 & 7.36 \\
\hline
\end{tabular}

Table 5. Mean TKW and grain yield of naked barley landraces over the years

Genotype

\begin{tabular}{|c|c|c|}
\hline \multirow[t]{2}{*}{ Genotype } & & TK \\
\hline & 2016 & 2 \\
\hline NGRC00837 & $31.89^{\mathrm{gh}}$ & \\
\hline NGRC00886 & $37.99^{\mathrm{bcd}}$ & \\
\hline NGRC02306 & $42.44^{\mathrm{a}}$ & \\
\hline NGRC02309 & $36.34^{\mathrm{def}}$ & 3 \\
\hline NGRC02312 & $27.95^{\mathrm{i}}$ & 33 \\
\hline NGRC02318 & $33.67^{\text {fgh }}$ & 34 \\
\hline NGRC02350 & $37.99^{\mathrm{bcd}}$ & 42 \\
\hline NGRC02357 & $39.99^{\mathrm{abc}}$ & 44 \\
\hline NGRC02363 & $37.14^{\text {cde }}$ & 40 \\
\hline NGRC04003 & $34.60^{\text {efg }}$ & 41 \\
\hline NGRC04894 & $40.43^{a b}$ & \\
\hline NGRC04902 & $42.73^{\mathrm{a}}$ & 4 \\
\hline NGRC04903 & $36.64^{\mathrm{de}}$ & \\
\hline NGRC06024 & $42.59^{\mathrm{a}}$ & \\
\hline NGRC06026 & $37.91^{\mathrm{bcd}}$ & 4 \\
\hline NGRC06034 & $37.43^{\text {cde }}$ & 3 \\
\hline NGRC06036 & $35.77^{\mathrm{def}}$ & 42. \\
\hline NGRC06042 & $31.04^{\mathrm{h}}$ & \\
\hline NGRC06043 & $31.12^{\mathrm{h}}$ & \\
\hline Solu Uwa & $32.22^{\mathrm{gh}}$ & \\
\hline $\mathrm{P}$ value & $<0.0001$ & $<0$ \\
\hline
\end{tabular}

$\mathrm{P}$ value

year×genotype

$\operatorname{LSD}(0.05)$

$\mathrm{CV}, \%$
TKW (g)

2017

Combined

$32.93^{\mathrm{h}} \quad 32.41^{\mathrm{hi}}$

$40.60^{\text {cdef }}$

$43.60^{\mathrm{abcd}}$

$37.33^{\mathrm{fg}}$

$33.63^{\mathrm{gh}}$

$34.13^{\text {gh }}$

$42.50^{\text {cde }}$

$44.20^{\mathrm{abc}}$

$40.53^{\text {cdef }}$

$41.87^{\text {cde }}$

$47.07^{\mathrm{ab}}$

$47.40^{\mathrm{a}}$

$38.97^{\text {ef }}$

$43.33^{\text {abcde }}$

$42.67^{\text {cde }}$

$39.77^{\text {def }}$

$42.76^{\text {bcde }}$

$30.83^{\mathrm{h}}$

$32.97^{\text {gh }}$

$39.50^{\mathrm{def}}$

$<0.0001$

2.928

4.87

$\begin{array}{rr}4.374 & 2.589 \\ 6.64 & 5.91\end{array}$

6.64

5.91 $39.30^{\text {de }}$

$43.02^{\mathrm{ab}}$

$36.84^{\text {ef }}$

$30.79^{\mathrm{i}}$

$33.90^{\text {gh }}$

$40.25^{\mathrm{cd}}$

$42.09^{\mathrm{bc}}$

$38.84^{\text {de }}$

$38.24^{\text {def }}$

$43.75^{\mathrm{ab}}$

$45.06^{\mathrm{a}}$

$37.80^{\text {def }}$

$42.96^{\mathrm{ab}}$

$40.29^{\mathrm{cd}}$

$38.60^{\text {de }}$

$39.27^{\text {de }}$

$30.94^{\mathrm{i}}$

$32.04^{\text {hi }}$

$35.86^{\mathrm{fg}}$

$<0.0001$

0.05
Grain yield (t/ha)

$2016 \quad 2017 \quad$ Combined

$2.30^{\text {bcde }} \quad 3.35^{\text {bc }} \quad 2.82^{\text {bcdefghi }}$

$2.23^{\text {bcdef }}$

$4.33^{\mathrm{a}}$

$3.28^{\mathrm{ab}}$

$2.28^{\text {bcdef }}$

$3.29^{\mathrm{bc}}$

$2.78^{\text {bcdefghi }}$

$2.60^{\mathrm{abc}} \quad 3.83^{\mathrm{ab}}$

$1.64^{\mathrm{f}} \quad 3.29^{\mathrm{bc}}$

$3.21^{\mathrm{abc}}$

$1.97^{\text {cdef }}$

$3.40^{\mathrm{bc}}$

$2.47^{\text {efghi }}$

$1.72^{\text {ef }}$

$2.69^{\text {cdefghi }}$

$2.68^{\mathrm{ab}} \quad 3.09^{\mathrm{bc}} \quad 2.89^{\mathrm{bcdef}}$

$1.73^{\text {ef }}$

$1.72^{\text {ef }}$

$2.66^{\mathrm{ab}}$

$2.91^{\mathrm{c}}$

$2.89^{\mathrm{c}}$

$3.36^{\mathrm{bc}}$

$2.32^{\text {ghi }}$

$3.11^{\mathrm{a}} \quad 3.81^{\mathrm{ab}}$

$2.30^{\text {hi }}$

$2.28^{\text {bcdef }} \quad 3.63^{\text {abc }}$

$3.01^{\mathrm{abcd}}$

$2.38^{\mathrm{bcd}}$

$3.32^{\mathrm{bc}}$

$2.52^{\mathrm{abc}}$

$3.67^{\mathrm{abc}}$

$2.36^{\text {bcde }} \quad 3.33^{\text {bc }}$

$2.35^{\text {bcde }}$

$2.83^{\mathrm{c}}$

$2.60^{\mathrm{abc}}$

$3.08^{\mathrm{bc}}$

$1.76^{\text {def }}$

$2.98^{\mathrm{bc}}$

$3.86^{\mathrm{ab}}$

0.1

0.001

$2.96^{\text {abcde }}$

$2.85^{\text {bcdefg }}$

$3.10^{\text {abcd }}$

$2.84^{\text {bcdefgh }}$

$2.59^{\text {defghi }}$

$2.84^{\mathrm{bcdefgh}}$

0.00

$2.37^{\text {fghi }}$

$3.15^{\mathrm{abc}}$

$<0.0001$

0.33 


\section{DISCUSSION}

Landraces are populations that farmers select based on their functional traits, manage over time through a sequence of cropping seasons and become adapted to their local environment (Bajracharya et al 2012). In the crops like naked barley, farmers have very few varietal options for cultivations in their field because only one released variety that is Solu uwa is available to the farmers. Farmers, however, preserved and continuously cultivated naked barley landraces in their field from generation to generations. About 340 local landraces of naked barley from different locations are preserved at NAGRC conservation facility. Landraces are recognized to present a tangible crop genetic resource of actual or potential economic benefit for humankind at national, regional and global levels (Chalak et al 2015). They provide a large gene pool for future genetic improvement programs and food security (Ceccarelli 1994) and are developed during long-term traditional cultivation at the same location where they are exposed to both human selection and eco-geographic pressures (Chalak et al 2015). They are adapted to fit in the environment of their location of origin while modern cultivars are developed for high yield and seldom targeted at a particular location (Gepts and Papa 2002, Lasa et al 2001).

Variability of quantitative traits of any crop is influenced by genetic factors, environmental factors and their interaction whereas uniformity of individuals and stability of quantitative traits are major requirements for the development of improved varieties and their release. Since the preservation of broad genetic base of landraces could be much appreciated, then the study of variability of quantitative traits becomes increasingly important (Chalak et al 2015). In the present study, the most variables traits were spikes per $\mathrm{m}^{2}(26.36 \%)$, plant height $(\mathrm{cm})(18.19 \%)$, grain yield $(\mathrm{t} / \mathrm{ha})(16.18 \%)$ and grain per spike $(7.36 \%)$ with respective value of $26.36 \%, 18.19 \%, 16.18 \%$ and $7.36 \%$ coefficient of variation. Such a strong variability was caused by the fact that these are complex traits, controlled by a polygenetic system and are strongly influenced by environmental factors. Chalak et al (2015) reported similar pattern of variability in barley landraces. Days to heading and days to maturity expressed the lowest variability with a coefficient of variation of $1.57 \%$ and $1.68 \%$ respectively and followed by thousand kernel weight $(5.91 \%)$ and grain per spike $(7.36 \%)$. These results match with the findings of Singh (2011), who reported that days to heading and days to maturity were the most stable traits, whereas yield and yield components were noticed for strong phenotypic and genotypic variability about $28 \%$.

Evaluation of landraces showed significant difference in functional traits like plant height, grain per spike, TKW, grain yield etc and also to the ACI value for yellow rust. Our study showed that naked barley genotypes NGRC04902, NGRC00886, NGRC02309 and NGRC06026 were high yielding among the tested genotypes. Ghimire et al (2019) also reported NGRC4902 and NGRC02309 as high yielding landraces in Khumaltar condition. Ghimire et al. (2019) stated landraces NGRC02306, NGRC04902 and NGRC04894 were the high yielding but NGRC04894 was found the most stable genotype with better adaptability whereas NGRC02306 and NGRC04902 were high yielding landraces adapted to high yielding environment namely Khumaltar. However, the lower yielding landrace NGRC02327 was the earliest and could be preferred by farmers as its maturity allows it to fit better in the rice based cropping system. Likewise, Yadav et al. (2018) also stated that naked barley landraces NGRC04894, Lamjung local, NGRC02306, NPGR1579, NGRC02327 and NPGR1579 exhibited morphological superiority and potentially for utilization as genitors in crop improvement programs.

In many cereal-rust patho-systems, the quantitative aspects of cultivar resistance have been described and estimated by means of average coefficient of infection (ACI) values for adult plant resistance (Broers et al 1996, Pathan and Park 2006). In our study NGRC00837 was found having the lowest ACI value of yellow rust than other tested genotypes which indicates this genotype was resistant to yellow rust diseases.

Significant responses of tested genotypes over growing years suggested the environmental influence on the expression of those traits (Adhikari et al 2018). However non significant interaction between 
genotypes $\mathrm{x}$ year were found on days to heading, days to maturity, plant height, grain per spikes, spike

per $\mathrm{m}^{2}$, TKW and grain yield, showed the stable performance among tested genotypes. In line with this findings, very similar reporting were made earlier by Jalata et al (2011). Likewise, significant interaction of genotypes $\mathrm{x}$ year was found only on ACI value for yellow rust diseases, that showed variable response of genotypes to yellow rust diseases over the years. Karki and Karki (1996) has also observed that yellow rust diseases of wheat is very sensitive to environmental changes and its occurrence is not very regular.

\section{CONCLUSIONS}

The present study concluded that the presence of significant genetic variability among the tested genotypes of naked barley. We found that genotypes NGRC04902, NGRC00886, NGRC02309 and NGRC06026 were high yielding; Genotypes NGRC02363, NGRCO06034, NGRC04903 and NGRC00837 were early maturing type; and genotype NGRC00837 was yellow rust resistant among the tested genotypes and these are elite genotypes for hill agriculture. Selected elite landraces need to be tested in farmer's field in larger areas followed by variety release or registration process which would help to diversify varietal options for the farmers. Hence, utilization of these better germplasm lines with desirable characteristics ie high yielding, diseases resistant, early maturity etc. in barley improvement program will help in breaking the yield plateau.

\section{ACKNOWLEDGEMENTS}

The financial support received from Nepal Agricultural Research Council is highly acknowledged.

\section{REFERENCES}

Adhikari BN, BP Joshi, J Shrestha and YK Joshi. 2018. Agronomic performance and correlation study in barley (Hordeum vulgare L.) genotypes. Scholars Journal of Agriculture and Veterinary Sciences 5(12):658-663.

Akar T, E Franci, A Tondelli, F Rizza, AM Stanca and N Pecchioni. 2009. Marker-assisted characterization of frost tolerance in barley (Hordeum vulgare L.). Plant Breeding 128(4): 381-386.

Bajracharya J, AHD Brown, BK Joshi, D Pandey, BK Baniya, BR Sthapit and DI Jarvis. 2012. Traditional seed management and genetic diversity in barley varieties in high-hill agro-ecosystems of Nepal. Genetic Resources and Crop Evolution 59:389-398. DOI: https://doi.org/10.1007/s10722-011-9689-2 .

Broers LHM, X Cuesta-Subias and RM Lopez-Atilano. 1996. Field assessment of quantitative resistance to yellow rust in ten spring bread wheat cultivars. Euphytica 90:9-16.

Ceccarelli S. 1994. Specific adaptation and breeding for marginal conditions. Euphytica 77: 205-219.

Chalak L, R Mzid, W Rizk, H Hmedeh, R Kabalan, J Breidy, B Hamadeh, H Machlab, H Rizk and S Elhajj. 1015. Performance of 50 Lebanese barley landraces (Hordeum vulgare L. subsp. vulgare) in two locations under rainfed conditions. Annals of Agricultural Science 60(2):325-334.

Chen XM, RF Line and H Leung. 1995. Virulence and polymorphic DNA relationships of Puccinia striiformis $\mathrm{f}$. sp. hordei to other rusts. Phytopathology 85:1335-1342.

Cross P and G Edwards-Jones. 2006. Variation in pesticide hazard from arable crop production in Great Britain from 1992 to 2002: pesticide risk indices and policy analysis. Crop Protection 25 (10):1101-1108.

Dubin HJ and RW Stubbs. 1986. Epidemic spread of barley stripe rust in S. A. Plant Diseases 70 (2):141-44.

Fischbeck G. 2003. Diversification through breeding. In: Diversity in barley (R Bothmer, T Hintum, H Knupffer and K Sato, eds). Elsevier, Amsterdam, pp. 29-52.

Foster-Powell K, SHA Holt, JC Brand-Miller. 2002. International table of glycemic index and glycemic load values. The American Journal of Clinical Nutrition 76 (1):5-56.

Genebank. 2018. Annual Report 2074/75 (2017/18). National agriculture genetic resources centre, NARC (KH Ghimire, D Singh and A Karkee eds.). Khumaltar, Lalitpur, Nepal.

Gepts P and R Papa. 2002. Encyclopedia of life sciences. In: Evolution during domestication. Nature Publishing Group, London, pp. 1-7.

Ghimire KH and RT Magar. 2017. Diversity in Nepalese wheat genetic resources as revealed by Agromorphological markers. Internatinoal Journal of Scientific and Engineering Research 8(7): 1646-1651.

Ghimire KH, BK Joshi, R Gurung, E Palikhey, N Pudasaini and A Parajuli. 2019. Adaptability of naked barley landraces in mountain agro-ecosystem of Nepal. Journal of Nepal Agric Research Council. 5:34-42. DOI: https://doi.org/10.3126/jnarc.v5i1.22064 
Ghimire KH, M Bhattarai, BK Joshi and MR Bhatta. 2013. Agro-Morphological Characterization of Nepalese Rice (Oryza sativa L.) Landraces. Proceedings of the $27^{\text {th }}$ National summer crop workshop, Nepal Agricultural Research council, Sinhadarbar plaza, Kathmandu.

Gomez KA and AA Gomez. 1984. Statistical procedures for agricultural research. New York: Wiley.

Gurung R, SR Sthapit, D Gauchan, BK Joshi and BR Sthapit. 2017. Baseline survey report: I. Ghanpokhara, Lamjung, Integrating traditional crop genetic diversity into technology: using a biodiversity portfolio approach to bufer against unpredictable environmental change in the Nepal Himalayas. Pokhara, Nepal: LI-BIRD, NARC, DOA and Bioversity International.

IPGRI. 1994. Descriptors for Barley (Hordeum vulgare L.). International Plant Genetic Resources Institute, Rome, Italy.

Jalata Z, A Ayana and H Zeleke. 2011. Variability, heritability and genetic advance for some yield and yield related traits in Ethiopian barley (Hordeum vulgare L) landraces and crosses. International Journal of Plant Breeding and Genetics 5(1):44-52.

Joshi BK, AK Acharya, D Gauchan and MR Bhatta. 2017a. Agrobiodiversity status and conservation options and methods. In: Conservation and Utilization of Agricultural Plant Genetic Resources in Nepal (BK Joshi, HB KC and AK Acharya, eds). Proceedings of 2nd National Workshop, 22-23 May 2017, Dhulikhel; NAGRC, FDD, DoA and MoAD; Kathmandu, Nepal; pp. 21-38.

http://moad.gov.np/public/uploads/855517450-Plant\%20Genetic\%20Resources_CUAPGR_Nepal-min.pdf

Joshi BK, MR Bhatta, KH Ghimire, M Khanal, SB Gurung, R Dhakal, and BR Sthapit. 2017b. Released and Promising Crop Varieties of Mountain Agriculture in Nepal (1959-2016). LI-BIRD, Pokhara; NARC, Kathmandu and Bioversity International, Pokhara, Nepal. https://www.bioversityinternational.org/fileadmin/user_upload/Released_and_promising_crop_varieties.pdf

Joshi BK, SB Gurung, PM Mahat, B Bhandari, and D Gauchan. 2018. Intra-Varietal Diversity in Landrace and Modern Variety of Rice and Buckwheat. The Journal of Agriculture and Development 19:1-8. https://cgspace.cgiar.org/handle/10568/97576

Kandel M, NB Dhami and J Shrestha. 2019. Performance evaluation of Barley (Hordeum vulgare L.) genotypes in Dolakha, Nepal: from yielding perspective. Journal of Agriculture and Natural Resources 2(1): 322-337.

Karki CB and PB Karki. 1996. Wheat disease report 1994-1951. In: Proceedings of wheat research reports, National Wheat Reserch Program, Bhairawa.

Lasa JM, E Igartua, FJ Ciudad, P Codesal, EV Garciaa, MP Gracia, B Medina, BI Romagosa, JL Molina-Cano and JL Montoya. 2001. Morphological and agronomical diversity patterns in the Spanish barley core collection. Hereditas 135:217-225.

Line RF. 2002. Stripe rust of wheat and barley in North America: a retrospective historical review. Annual Review of Phytopathology 40:75-118.

Marshall D and RL Sutton. 1995. Epidemiology of stripe rust, virulence of Puccinia striiformis f. sp. hordei and yield loss in barley. Plant Disease 79:732-737.

Palikhey E, SR Sthapit, S Gautam, D Gauchan, BK Joshi and BR Sthapit. 2017. Baseline survey report: III. Haku, Jumla. Integrating traditional crop genetic diversity into technology: using a biodiversity portfolio approach to bufer against unpredictable environmental change in the Nepal Himalayas. Pokhara, Nepal: LI-BIRD, NARC, DOA and Bioversity International.

Pandey M, D Kopahnke, AHabekuss, W Friedt and F Ordon. 2009. Screening Nepalese hulless barley germplasm for resistance to major fungal and viral diseases. Journal of the Institute of Agriculture and Animal Sciences 30:115-124.

Parajuli A, A Subedi, SR Sthapit, D Gauchan, AAdhikari, BK Joshi and Sthapit. 2017. Baseline survey report: IV. Chhipra, Humla, integrating traditional crop genetic diversity into technology: using a biodiversity portfolio approach to bufer against unpredictable environmental change in the Nepal Himalayas. Pokhara, Nepal: LI-BIRD, NARC, DOA and Bioversity International.

Pathan AK and K Park. 2006. Evaluation of seedling and adult plant resistance to leaf rust in European wheat cultivars. Euphytica 149:327-342.

Peterson RF, AB Campbell and AE Hannah. 1948. A diagrammatic scale for estimating rust intensity of leaves and stems of cereals. Canadian Journal of Research 26 (6):496-500.

Pudasaini N, SR Sthapit, D Gauchan, B Bhandari, BK Joshi and BR Sthapit. 2017. Baseline survey report: I. Ghanpokhara, Lamjung. Integrating traditional crop genetic diversity into technology: using a biodiversity portfolio approach to buffer against unpredictable environmental change in the Nepal Himalayas. Pokhara, Nepal: LI-BIRD, NARC, DOA and Bioversity International.

Ragaee S, ES Abdel-Aal and M Noaman. 2006. Antioxidant activity and nutrient composition of selected cereals for food use. Food Chemistry 98(1):32-38.

Roelfs AP, RP Singh and S Saari. 1992. Rust diseases of wheat: concepts and methods of diseases management. Mexico: CIMMYT. p. 81. 
Selvakumar R, RPS Verma, MS Saharan, SC Bhardwaj, PS Shekhawat, M Meetajingal, D Singh, R Devlash, SS Karwasra, SK Jain and I Sharma. 2015. Seedling and adult plant resistance of barley genotypes to stripe rust pathogen (Puccinia striiformis f. sp. hordei). Indian Phytopatholoty 68 (2): 218-220.

Shrestha J. 2019. P-Value: A true test of significance in agricultural research. https://www.linkedin.com/pulse/pvalue-test-significance-agricultural-research-jiban-shrestha/.

Singh AP. 2011. Genetic variability in two-rowed barley (Hordeum vulgare L.). Indian Journal of Scientific Research 2:22-23.

Taketa S, S Kikuchi, T Awayama, S Yamamoto, M Ichii and S Kawasaki. 2008. Monophyletic origin of naked barley inferred from molecular analyses of a marker closely linked to the naked caryopsis gene (nud). Theoretical and Applied Genetics 108:1236-1242.

Valkoun J and J Konopka. 2004. Global inventory of barley genetic resources. In: Proceedings of the 9th International Barley Genetics Symposium. Brno, Czech Republic; p. 31-8.

Wood PJ. 2002. Relationships between solution properties of cereal b-glucans and physiological effects-a review. Trends in Food Science and Technology 13:313-320.

Yadav RK, S Gautam, E. Palikhey, BK Joshi, KH Ghimire, R Gurung, AR Adhikari, N Pudasaini and R Dhakal. 2018. Agro-morphological diversity of Nepalese naked barley landraces. Agriculture and Food Security 7:86.

Zohary D and M Hopf. 2000. Domestication of plants in the old world. Oxford University Press, Oxford.

$|1-----|-1|-----| \mid$ 